\title{
Editorial
}

\section{Acknowledgement to Reviewers of Antioxidants in 2013}

Antioxidants Editorial Office

MDPI AG, Klybeckstrasse 64, CH-4057 Basel, Switzerland

Published: 28 February 2014

The editors of Antioxidants would like to express their sincere gratitude to the following reviewers for assessing manuscripts in 2013:

Abbas, S.

Abdel-Aal, El-sayed M.

Alves, Andreia

Andini, Rita

Andreotti, Carlo

Apak, Reşat

Aruoma, Okezie

Baiano, A.

Barrajón-Catalán, Enrique

Battino, Maurizio

Benavente-Garcia, O.

Bendini, Alessandra

Böhm, Volker

Brambilla, A.

Brantner, Adelheid

Calokerinos, Anthony

Carvalho, Paulo

Celi, Pietro

Céspedes, Carlos L.

Chen, Ching-Yi

Choi, Young Hae

Clifford, Mike

Coelho, José A.

Dalessandro, Giuseppe
De Bellis, Luigi

De Tullio, M.C.

Dean, Lisa

Durazzo, Alessandra

Espley, Richard V.

Estevinho, Leticia

Failla, Mark

Farid, Chemat

Franco, Rodrigo

Fry, Jeffery

Giannenas, I.

Gordon, Michael

Goya, Luis

Hadjipavlou-Litina, Dimitra

Haleagrahara, Nagaraja

Harris, Cory

Innocenti, Marzia

Iriti, Marcelo

Jastrebova, Jelena

Kaiser, Andrea

Khan, M. Firoze

Khandaker, Laila

Kremer, Dario
Liakopoulou-Kyriakides,

Maria

Liu, Wenlan

Loizzo, Monica R.

Ludewig, Gabriele

Malathy, P.

Malta, Luciana Gomes

Medina, Miguel A'ngel

Miceli, Antonio

Miguel, M. G.

Miller, Kenneth B

Mimica-Dukic, Neda

Ming, Li-june

Morales, Juan C.

Mosley, Robert Lee

Mullen, Bill

Nemzer, Boris

Niki, Etsuo

Özyürek, Mustafa

Paliyath, Gopinadhan

Panzella, Lucia

Pérez-Jiménez, Jara

Pizzala, Roberto

Polidoros, Alexios 
Polikarpov, Igor

Priefer, Ronny

Proestos, Charalampos

Pyrzynska, Krystyna

Ragaee, S. M.

Rahman, Anisur

Romain, Cindy

Romano, Anabela

Rouanet, Jean-Max

Roychowdhury, Sanjoy

Ruiz-Sanz, José Ignacio

Rupasinghe, H.P. Vasantha
Ryan, Michael J.

Saleh, Mahmoud A.

Salvador, Mirian

Sarker, Satya

Sato, Kenji

Saul, Nadine

Schmeda Hirschmann,

Guillermo

Senchina, David

Serrano, María

Sommano, Sarana

Spanier, Britta
Stevanovic, Tatjana

Stich, Karl

Striker, Gary E.

Sureda, Antoni

Tundis, Rosa

Udenigwe, Chibuike C.

Ulrichová, Jitka

Vadigepalli, Rajanikanth

Vieira, A.

Weber, John T.

Williams, Robert J.

Zhang, Junzeng

(C) 2014 by the authors; licensee MDPI, Basel, Switzerland. This article is an open access article distributed under the terms and conditions of the Creative Commons Attribution license (http://creativecommons.org/licenses/by/3.0/). 